\title{
Immunotherapy in EGFR mutant non-small cell lung cancer: when, who and how?
}

\author{
Debora Bruno, Afshin Dowlati \\ Division of Hematology and Oncology, Department of Medicine, University Hospitals Seidman Cancer Center and Case Western Reserve \\ University, Cleveland, Ohio, USA \\ Correspondence to: Afshin Dowlati, MD. University Hospitals, 11100 Euclid Avenue, Cleveland, Ohio 44106, USA. Email: Afshin.dowlati@case.edu. \\ Provenance: This is an invited article commissioned by the Guest Section Editor Tao Shi (Clinical Cancer Institute of Nanjing University, \\ Nanjing, China). \\ Comment on: Yang JC, Gadgeel SM, Sequist LV, et al. Pembrolizumab in Combination With Erlotinib or Gefitinib as First-Line Therapy for \\ Advanced NSCLC With Sensitizing EGFR Mutation. J Thorac Oncol 2019;14:553-9.
}

Submitted May 31, 2019. Accepted for publication Jun 05, 2019.

doi: $10.21037 /$ tlcr.2019.06.02

View this article at: http://dx.doi.org/10.21037/tlcr.2019.06.02

Mutations in the epidermal growth factor receptor (EGFR) are detected in approximately $15 \%$ of lung adenocarcinomas in western countries, with even higher rates noted in Asian patients. The most common alterations that lead to constitutive activation of its tyrosine kinase are in-frame deletions of exon 19 and the L858R mutation in exon 21. Single-agent tyrosine kinase inhibitor (TKI) therapy leads to substantial progression-free survival (PFS) benefit with very manageable toxicity profiles. However, resistance ultimately ensues, including development of a second EGFR mutation such as T790M (seen in $60 \%$ of patients who progress after first or second generation TKIs), acquisition of mutations in other receptors (MET) or transformation to more aggressive histology such as small cell lung cancer (approximately $5 \%$ of cases). When resistance develops, patients are treated with systemic chemotherapy and ultimately succumb to the disease, hence the need for more effective therapies.

A small percentage of non-small cell lung cancer (NSCLC) patients derive a prolonged benefit from checkpoint inhibitor therapy, with most recent updated analysis of phase 1 trials with checkpoint inhibitor nivolumab demonstrating a $16 \% 5$-year overall survival (OS) in patients with metastatic disease. Although the presence of driver oncogenic mutations predicts high response rates to TKI therapy, predictive biomarkers are not as robust for immune-oncology (IO) agents. The only currently FDAaccepted discriminator of potential benefit of first line single agent program death 1 (PD-1) inhibitor pembrolizumab for metastatic NSCLC remains PD-ligand 1 (PD-L1) expression measured as $50 \%$ or greater tumor cells staining positive for PD-L1 (TPS $\geq 50 \%$ ).

Other potential biomarkers have been pursued and are currently under investigation in clinical trials. Both melanoma and NSCLC, the anointed malignancies with the higher success rate of checkpoint inhibitor therapy, are the epitomes of malignancies with high rates of cumulative somatic mutations due to carcinogen exposure (UV light and tobacco smoke). Higher nonsynonymous mutation burden (TMB) in NSCLC correlated with response, PFS and duration of response (DOR) to pembrolizumab (1). Most of such mutations occur in "passenger genes", leading to the expression of various peptides (tumor neoantigens) which are completely novel to the organism's proteome and as such able to elicit a more potent activation of effector $\mathrm{T}$ cells. In fact, NSCLC tumors harboring at least 10 mutations per megabase were more likely to respond ( $45 \% \mathrm{RR}$ ) and remain responsive to dual checkpoint inhibitor therapy targeting PD-1 and cytotoxic T-lymphocyte associated antigen 4 (CTLA-4) when compared to cytotoxic chemotherapy in the phase III CheckMate 227 trial, regardless of tumor's PD-L1 expression (2).

Patients harboring tumors with driving mutations such as EGFR exon19 del/L858R and ALK-rearrangement have notoriously failed to benefit from IO therapy in the initial phase III trials looking at single agent checkpoint inhibitors 
versus docetaxel (3-5), with retrospective and meta-analyses confirming this finding $(6,7)$. This has led to the exclusion of such patient cohort from most subsequent studies. Many are the hypotheses to explain such discouraging results.

PD-L1 expression by EGFR mutated tumors has actually been described as a mechanism for immune evasion in preclinical studies (8). Pre-clinical data demonstrates activation of EGFR through EGF or activating mutations exon19 del and L858R in vitro leading to PD-L1 overexpression by tumor cells through the ERK1/2-c-jun pathway. This phenotype is capable of inducing T-cell apoptosis through PD-1/PD-L1 axis in coculture systems (9). Treatment with gefitinib downregulates PD-L1 expression and re-engages co-cultured T-cells. As expected, combining anti-PD1 and TKI therapies does not lead to in vitro synthetic lethality. The available clinical data though is discordant in regards to PD-L1 expression in EGFR mutated NSCLC $(10,11)$, with two meta-analyses failing to confirm a positive correlation with EGFR mutations $(12,13)$. Such differences may reflect types of specimens obtained (such as pre- versus post-TKI exposure) as well as differences in the technical aspects of PD-L1 testing.

EGFR mutated tumors generally have lower TMB than EGFR wild-type tumors, however EGFR mutated tumors that do have high TMB values seem to have worse outcomes with TKI therapy, with shorter time to discontinuation of TKI as well as OS (14). Lower TMB correlates with decreased production of neoantigens and hence an increased diversity of T-cell receptor repertoire (15) leading to less clonal expansion.

In this current study, Yang and colleagues (16) explore the feasibility of combining erlotinib and gefitinib with pembrolizumab in patients with EGFR-mutant NSCLC enrolled in cohorts E and F of KEYNOTE-021. KEYNOTE-021 trial (NCT02039674) is a multi-cohort phase $1 / 2$ trial investigating the feasibility and the activity of pembrolizumab in combination with chemotherapy, TKI therapy or immunotherapy (CTLA4 inhibitor ipilimumab) as first line therapy in patients with stage IIIB and metastatic NSCLC, regardless of PD-L1 expression. In this phase 1/2 study with sequential $3+3$ enrollment of up to 12 patients in each group, followed by cohort expansion based on dose limiting toxicities, standard daily doses of TKIs (erlotinib $150 \mathrm{mg}$ in cohort $\mathrm{E}$ and gefitinib $250 \mathrm{mg}$ in cohort $\mathrm{F}$ ), were utilized in combination with $2 \mathrm{mg} / \mathrm{kg}$ pembrolizumab IV every 3 weeks. Oral TKI therapy would be continued as long as patients derived clinical benefit but pembrolizumab would be discontinued after 2 years. The primary endpoint was dose finding for further evaluation in phase 2 , with planned secondary analysis of DOR, PFS, and OS.

While no DLTs or grade 5 AEs occurred in either cohort, enrollment in the gefitinib group was discontinued after $5 / 7(71.4 \%)$ patients developed grade 3 or 4 AST/ ALT elevations. All cases of transaminitis occurred outside the defined period for DLT (greater than the first 3-week treatment cycle), required the use of steroids for greater than 21 days and led to treatment discontinuation. The median number of pembrolizumab infusions in this group was 3 . The combination was therefore deemed not feasible and that cohort did not meet the primary endpoint of the study.

In cohort $\mathrm{E}$, the combination was deemed feasible, with most common toxicities observed being rash (6/12-one of them grade 3 ) and diarrhea (4/12), with no grade 4 AEs. The most common auto-immune side effects consisted of hypothyroidism (4/12) and severe skin reactions (2/12, grade 3). Interestingly, one patient developed neuralgic amyotrophy coupled with LFT elevation (both grade 3) within 3 weeks of treatment initiation. The median number of pembrolizumab infusions in the erlotinib group was 18 .

In regards to efficacy and potential predictor effects of biomarker PD-L1 expression, the two cohorts had very different representations. Of the patients enrolled in the gefitinib group, $85.7 \%(6 / 7)$ had PD-L1 TPS $<1 \%$, while $83.3 \%$ of the patients in the erlotinib cohort had PD-L1 TPS $>1 \%$, with $4 / 12(33.3 \%)$ scoring $\geq 50 \%$. Still, the ORR observed in the erlotinib group was $41.7 \%$ (much lower than historical controls using single agent erlotinib in first line setting). However, all of the patients (4/4) with PD-L1 TPS $\geq 50 \%$ responded to treatment, and of those, 3 out of 4 remain responsive and 1 out of 4 achieved 18.3 months of DOR. The median PFS in the erlotinib cohort was 19.5 months. The ORR in the gefitinib group was $14.3 \%$. The subtypes of EGFR mutations of the patients enrolled in this study were not reported.

Safety concerns regarding the combination of EGFR TKIs and checkpoint inhibitors have been raised in previous studies. In a trial combining third generation TKI osimertinib with PD-L1 inhibitor durvalumab in treatment naïve patients with EGFR mutations, ALT elevation was described in $65 \%$ of the patients with grade 3/4 AST/ALT elevation leading to treatment discontinuation in $30 \%$ of patients who were treated with osimertinib for 4 weeks prior to first dose of durvalumab (17). The overall RR was compatible with historical controls from phase 3 trials of single agent osimertinib in TKI naïve patients. PFS was not reported. A similar trial looking at this combination in both TKI naïve and experienced patients drew attention to a 
$38 \%$ rate of ILD (majority < grade 3/4 with no grade 5) (18). In a phase I trial exploring safety of $1 \mathrm{~L}$ erlotinib and PDL1 inhibitor atezolizumab however, though there was a frequency of $50 \%$ of serious AEs, only $7 \%$ developed grade 3/4 ALT elevation (19). Despite the very small number of patients enrolled in Cohort G of KEYNOTE-021, a 70\% incidence of grade 3/4 AST/ALT elevation is surprising, especially when compared to $5 \%$ of grade $3 / 4$ hepatotoxicity across multiple trials with single agent gefitinib. It is though conceivable that the hepatic toxicity displayed by the combination of gefitinib and pembrolizumab in this study is due to population pharmacokinetics. All participants in the gefitinib cohort were Asian, while 75\% (9/12) patients in the erlotinib group were White. Details regarding ethnicity of patients enrolled in other phase I trials evaluating the safety of such combination are not readily available.

When evaluating the responses and PFS provided in the study, we must focus on Cohort E-as it provided data on 12 treated patients. Overall, as a group, a $41.7 \%$ response was noted in the TKI-naïve patients treated with combination erlotinib and pembrolizumab, which is a disappointing rate. It also certainly does not compare favorably with previous phase I study looking at erlotinib in combination with atezolizumab, where a $75 \% \mathrm{RR}(\mathrm{n}=20)$ was noted (19), albeit we are comparing very small samples. Even more challenging it is to interpret the significance of $100 \%$ RR in four subjects in the subgroup TPS $\geq 50 \%$, due to such small numbers and especially due to the fact that most pts in cohort $\mathrm{E}$ had some degree of TPS $>1 \%$. The data available is still very immature to generate any hopes towards meaningful PFS.

Certainly the rationale to combine a TKI with IO therapy in treatment naïve patients could be justified based on pre-clinical and clinical data demonstrating an immunomodulatory effect of TKI therapy. For instance, both gefitinib and erlotinib have been demonstrated to promote immune response by enhancing NK cell cytotoxicity (20). A study analyzing peripheral blood samples of patients on gefitinib therapy for 4 weeks demonstrated a significant increase in the number of NK cells and IFN-gamma levels, as well as a decline in IL-6. In this particular study, tumor samples obtained after gefitinib therapy demonstrated downregulation of tumor cell PD-L1 expression mediated by TKI therapy (21). However, to this date, multiple early phase trials-including the current one being discussed-have failed to provide a substantial signal that enforces this combination as one suitable to pursuit both from an efficacy and a safety standpoint.
Therefore, many questions still remain unsolved on how we can introduce the concept of immune modulation in treating EGFR mutant NSCLC. When should we investigate IO therapy in such patients: in treatment naïve setting or following TKI failure? Who could potentially benefit: PD-L1 TPS $>50 \%$ only, specific EGFR mutated subtypes? How could we modulate the immune response in this population: will checkpoint inhibitor therapy alone as an IO agent suffice or should combination therapy be used?

To this time, evidence stands against the use of single agent checkpoint inhibitor in both treatment naïve and TKI failure EGFR patients, despite PD-L1 expression. The largest experience published to date on single agent pembrolizumab in TKI naïve EGFR mutant patients is discouraging. In a phase 2 trial, despite very high levels of PD-L1 expression- $73 \%$ of patients had TPS $\geq 50 \%$, enrollment was discontinued after 11 patients due to lack of efficacy (22).

So far the only positive clinical trial data on efficacy of IO therapy for both EGFR mutant and ALK rearrangement tumors has come from the IMPower 150 trial that combined cytotoxic agents, an anti-angiogenic drug and PD-L1 inhibition, in the setting of TKI failure. The phase III IMPower 150 trial randomized patients with TKI-failure EGFR and ALK-rearrangements to one of three groups: atezolizumab/carboplatin/paclitaxel (ACP), carboplatin/ paclitaxel/bevacizumab (BCP) or atezolizumab/carboplatin/ paclitaxel/bevacizumab (ABCP) (23). Secondary analyses of this EGFR/ALK mutated cohort demonstrated a statistically significant difference in PFS of 9.7 versus 6.1 months for the ABCP group (unstratified HR of 0.59) compared to the BCP group. No significant difference in OS was noted between ACP and BCP arms. Combining VEGF inhibition and TKI therapy in EGFR mutant NSCLC may overcome both primary and acquired resistance, with beneficial effects in PFS when bevacizumab is added to erlotinib in the first line setting. Bevacizumab administration, in combination with cytotoxic chemotherapy in patients with metastatic melanoma has been demonstrated to increase circulating number of CD8+ T cells, as well as decrease IL-6 levels, thus capable of immune modulation (24). Combining cytotoxic therapy with anti-angiogenic bevacizumab thus could have possibly permutated the "cold" tumor microenvironment typically seen in EGFR mutant tumors into a TIL enriched one. Neoadjuvant paclitaxel has been demonstrated capable of increasing number of TIL in breast tumors, with most notable effects in tumors undergoing complete clinical responses (25). It is plausible that release 
of neoantigens by apoptotic cells above a certain threshold could lead to sufficient DC uptake and presentation to T-cells with its subsequent activation.

We commend the efforts of the authors in exploring the feasibility of dual TKI and PD-1 inhibition in TKI-naïve patients with EGFR mutant NSCLC. Though combining erlotinib and pembrolizumab is feasible, there were no clear signals that this may be an effective strategy that should be pursued further. Perhaps as we move forward in future attempts to introduce the concept of IO to EGFR mutant NSCLC, we should focus on patients who failed TKI therapy, and attempt to revert the "cold tumor microenvironment" phenotype using different and creative strategies of immune modulation. Few interesting potential tactics are currently being investigated in the clinical trial arena, such as the phase I/II study exploring the combination of nivolumab with the cytotoxic/immunomodulatory drug plinabulin [NCT02846792], after TKI failure. Certainly though, the last chapter on immune modulation in EGFR mutant NSCLC has not yet been written.

\section{Acknowledgments}

None.

\section{Footnote}

Conflicts of Interest: The authors have no conflicts of interest to declare.

\section{References}

1. Rizvi NA, Hellmann MD, Snyder A, et al. Cancer immunology. Mutational landscape determines sensitivity to PD-1 blockade in non-small cell lung cancer. Science 2015;348:124-8.

2. Hellmann MD, Ciuleanu TE, Pluzanski A, et al. Nivolumab plus Ipilimumab in Lung Cancer with a High Tumor Mutational Burden. N Engl J Med 2018;378:2093-104.

3. Borghaei H, Paz-Ares L, Horn L, et al. Nivolumab versus Docetaxel in Advanced Nonsquamous Non-Small-Cell Lung Cancer. N Engl J Med 2015;373:1627-39.

4. Herbst RS, Baas P, Kim DW, et al. Pembrolizumab versus docetaxel for previously treated, PD-L1positive, advanced non-small-cell lung cancer (KEYNOTE-010): a randomised controlled trial. Lancet 2016;387:1540-50.
5. Rittmeyer A, Barlesi F, Waterkamp D, et al. Atezolizumab versus docetaxel in patients with previously treated non-small-cell lung cancer (OAK): a phase 3, openlabel, multicentre randomised controlled trial. Lancet 2017;389:255-65.

6. Gainor JF, Shaw AT, Sequist LV, et al. EGFR Mutations and ALK Rearrangements Are Associated with Low Response Rates to PD-1 Pathway Blockade in Non-Small Cell Lung Cancer: A Retrospective Analysis. Clin Cancer Res 2016;22:4585-93.

7. Lee CK, Man J, Lord S, et al. Checkpoint Inhibitors in Metastatic EGFR-Mutated Non-Small Cell Lung Cancer-A Meta-Analysis. J Thorac Oncol 2017;12:403-7.

8. Akbay EA, Koyama S, Carretero J, et al. Activation of the PD-1 pathway contributes to immune escape in EGFRdriven lung tumors. Cancer Discov 2013;3:1355-63.

9. Chen N, Fang W, Zhan J, et al. Upregulation of PDL1 by EGFR Activation Mediates the Immune Escape in EGFR-Driven NSCLC: Implication for Optional Immune Targeted Therapy for NSCLC Patients with EGFR Mutation. J Thorac Oncol 2015;10:910-23.

10. D'Incecco A, Andreozzi M, Ludovini V, et al. PD-1 and PD-L1 expression in molecularly selected non-small-cell lung cancer patients. Br J Cancer 2015;112:95-102.

11. Takada K, Okamoto T, Shoji F, et al. Clinical Significance of PD-L1 Protein Expression in Surgically Resected Primary Lung Adenocarcinoma. J Thorac Oncol 2016;11:1879-90.

12. Zhang $M$, Li G, Wang $Y$, et al. PD-L1 expression in lung cancer and its correlation with driver mutations: a metaanalysis. Sci Rep 2017;7:10255.

13. Lan B, Ma C, Zhang C, et al. Association between PD-L1 expression and driver gene status in non-small-cell lung cancer: a meta-analysis. Oncotarget 2018;9:7684-99.

14. Offin M, Rizvi H, Tenet M, et al. Tumor Mutation Burden and Efficacy of EGFR-Tyrosine Kinase Inhibitors in Patients with EGFR-Mutant Lung Cancers. Clin Cancer Res 2019;25:1063-9.

15. Miyauchi E, Matsuda T, Kiyotani K, et al. Significant differences in $\mathrm{T}$ cell receptor repertoires in lung adenocarcinomas with and without EGFR mutations. Cancer Sci 2018.

16. Yang JC, Gadgeel SM, Sequist LV, et al. Pembrolizumab in Combination With Erlotinib or Gefitinib as First-Line Therapy for Advanced NSCLC With Sensitizing EGFR Mutation. J Thorac Oncol 2019;14:553-9.

17. Gibbons DL, Chow LQ, Kim DW, et al. Efficacy, safety and tolerability of MEDI1436 (durvalumab [D]), a human 
IgG1 anti-programmed cell death-ligand 1 (PD-L1) antibody, combined with gefitinib (G): A phase I expansion in TKI-naive patients (pts) with EGFR mutant NSCLC. J Thorac Oncol 2016;11:S79-S91.

18. Ahn MJ, Yang J, Yu H, et al. Osimertinib combined with durvalumab in EGFR-mutant non-small cell lung cancer: results from the TATTON phase $1 \mathrm{~b}$ trial. J Thorac Oncol 2016;11:S115.

19. Ma BB, Rudin RC, Cervantes A, et al. Preliminary safety and clinical activity of erlotinib plus atezolizumab from a Phase Ib study in advanced NSCLC. Ann Oncol 2016. DOI: $10.1093 / \mathrm{annonc} / \mathrm{mdw} 594.005$.

20. Kim H, Kim SH, Kim MJ, et al. EGFR inhibitors enhanced the susceptibility to NK cell-mediated lysis of lung cancer cells. J Immunother 2011;34:372-81.

21. Sheng J, Fang W, Liu X, et al. Impact of gefitinib in early stage treatment on circulating cytokines and lymphocytes

Cite this article as: Bruno D, Dowlati A. Immunotherapy in EGFR mutant non-small cell lung cancer: when, who and how? Transl Lung Cancer Res 2019;8(5):710-714. doi: 10.21037/ tlcr.2019.06.02 for patients with advanced non-small cell lung cancer. Onco Targets Ther 2017;10:1101-10.

22. Lisberg A, Cummings A, Goldman JW, et al. A Phase II Study of Pembrolizumab in EGFR-Mutant, PD-L1+, Tyrosine Kinase Inhibitor Naive Patients With Advanced NSCLC. J Thorac Oncol 2018;13:1138-45.

23. Socinski MA, Jotte RM, Cappuzzo F, et al. Atezolizumab for First-Line Treatment of Metastatic Nonsquamous NSCLC. N Engl J Med 2018;378:2288-301.

24. Mansfield AS, Nevala WK, Lieser EA, et al. The immunomodulatory effects of bevacizumab on systemic immunity in patients with metastatic melanoma. Oncoimmunology 2013;2:e24436.

25. Demaria S, Volm MD, Shapiro RL, et al. Development of tumor-infiltrating lymphocytes in breast cancer after neoadjuvant paclitaxel chemotherapy. Clin Cancer Res 2001;7:3025-30. 